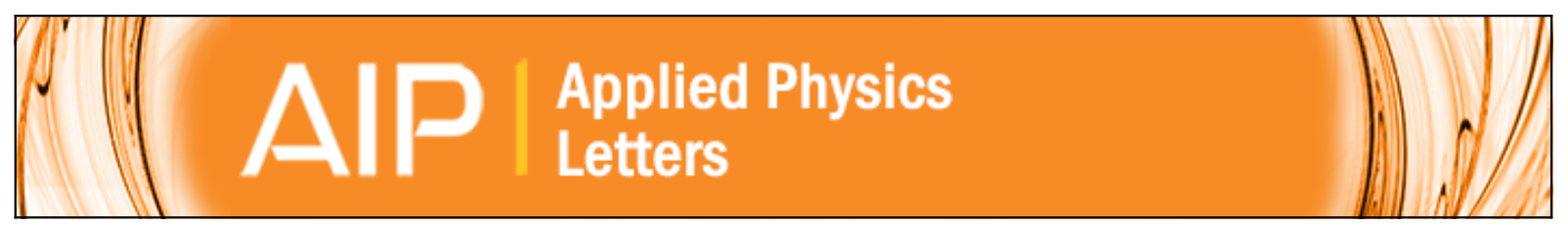

\title{
Zinc oxide integrated area efficient high output low power wavy channel thin film transistor
}

A. N. Hanna, M. T. Ghoneim, R. R. Bahabry, A. M. Hussain, and M. M. Hussain

Citation: Applied Physics Letters 103, 224101 (2013); doi: 10.1063/1.4836235

View online: http://dx.doi.org/10.1063/1.4836235

View Table of Contents: http://scitation.aip.org/content/aip/journal/apl/103/22?ver=pdfcov

Published by the AIP Publishing

\section{Articles you may be interested in}

Compositional tuning of atomic layer deposited $\mathrm{MgZnO}$ for thin film transistors

Appl. Phys. Lett. 105, 202109 (2014); 10.1063/1.4902389

Effect of Al concentration on Al-doped $\mathrm{ZnO}$ channels fabricated by atomic-layer deposition for top-gate oxide thin-film transistor applications

J. Vac. Sci. Technol. B 32, 041202 (2014); 10.1116/1.4880823

Atomic layer deposited zinc tin oxide channel for amorphous oxide thin film transistors

Appl. Phys. Lett. 101, 113507 (2012); 10.1063/1.4752727

Cycle time effects on growth and transistor characteristics of spatial atomic layer deposition of zinc oxide J. Vac. Sci. Technol. A 30, 01A154 (2012); 10.1116/1.3670878

High performance thin film transistor with low temperature atomic layer deposition nitrogen-doped $\mathrm{ZnO}$ Appl. Phys. Lett. 91, 183517 (2007); 10.1063/1.2803219

You don't

still use this

cell phone

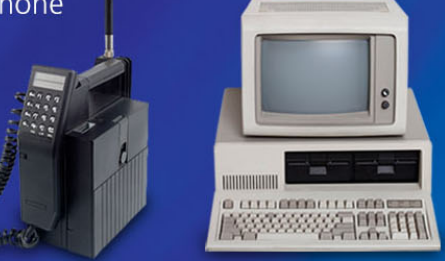

Why are you still using an AFM designed in the 80 's?
It is time to upgrade your AFM

Minimum \$20,000 trade-in discount for purchases before August 31st

Asylum Research is today's technology leader in AFM 


\title{
Zinc oxide integrated area efficient high output low power wavy channel thin film transistor
}

\author{
A. N. Hanna, M. T. Ghoneim, R. R. Bahabry, A. M. Hussain, and M. M. Hussain ${ }^{\text {a) }}$ \\ Integrated Nanotechnology Lab, Electrical Engineering, Computer Electrical Mathematical Science and \\ Engineering, King Abdullah University of Science and Technology, Thuwal 23955-6900, Saudi Arabia
}

(Received 25 August 2013; accepted 11 November 2013; published online 26 November 2013)

\begin{abstract}
We report an atomic layer deposition based zinc oxide channel material integrated thin film transistor using wavy channel architecture allowing expansion of the transistor width in the vertical direction using the fin type features. The experimental devices show area efficiency, higher normalized output current, and relatively lower power consumption compared to the planar architecture. This performance gain is attributed to the increased device width and an enhanced applied electric field due to the architecture when compared to a back gated planar device with the same process conditions. (C) 2013 AIP Publishing LLC. [http://dx.doi.org/10.1063/1.4836235]
\end{abstract}

Thin film transistor (TFT) is intended to produce high output current with lower power consumption. Since TFTs used in high resolution and large panel displays are in high demand from the consumers, low-cost integration of TFTs is critical. In accordance with the scaling trend of logic transistors, scaling the TFTs is also a pursued method to increase the output current. ${ }^{1}$ However, scaling is expensive, and it also shortens the width of the transistor. Therefore, a fin type feature integrated wavy channel architecture can play critical role to increase the width without increasing the transistor area and consequently can increase the output current. ${ }^{2}$ In this letter, we use atomic layer deposition (ALD) based zinc oxide $(\mathrm{ZnO})$ channel material (comparatively low cost oxide material with conformal and uniform deposition feature) to examine the effects of fin aspect ratio and pitch on the output current. In the recent past, semiconductor industry has started to use an advanced non-planar 3D Fin Field Effect Transistor (FinFET) or tri-gate device architecture for tighter electrostatic control over the channel and for mitigated Short Channel Effects (SCEs). ${ }^{3,4}$ In these devices charge transport takes place in the fin itself which are typically formed by patterning the substrate itself (most often a silicon-on-insulator substrate). Since we are using thin film based channel material therefore we pattern the substrate to use the fin as a mold for both the gate dielectric and the semiconductor layer in order to achieve an area efficient TFT. Since both channel uniformity and conformity are critical for the performance of our architecture, we used ALD for both of them. ${ }^{5}$ We chose $\mathrm{ZnO}$ as active material since amorphous oxide semiconductors (AOSs) are desirable for their high mobility, transparency, and low temperature deposition that allows integration on flexible substrates. ${ }^{6}$ We also chose high- $\kappa$ dielectric aluminum oxide $\left(\mathrm{Al}_{2} \mathrm{O}_{3}\right)$ as it forms a better interface with $\mathrm{ZnO}$ compared to conventional $\mathrm{SiO}_{2}{ }^{7}$

The process flow to fabricate the wavy channel TFT is as shown in Figs. 1(a)-1(d), where the fin features are first patterned in a heavily doped n-type silicon substrate, with a minimum resistivity of $0.008 \Omega \mathrm{cm}$, which is also used as a back gate. Fin features were etched using Deep Reactive Ion

\footnotetext{
${ }^{\text {a) }}$ Author to whom correspondence should be addressed. Electronic mail: muhammadmustafa.hussain@kaust.edu.sa
}

Etching Process (DRIE) process for 6 cycles. The fin height was measured to be $1.5 \mu \mathrm{m}$ by a profilometer. A planar control sample, without fins, was also processed in parallel with the exact same materials and process conditions and the planar device consumes the same chip area as the fin devices. The fin feature patterning process is followed by deposition of $50 \mathrm{~nm}$ of $\mathrm{ALD} \mathrm{Al}_{2} \mathrm{O}_{3}$ at $300^{\circ} \mathrm{C}$. Source and drain of titanium based adhesion layer followed by gold as metal contact (Ti-Au) were then deposited at room temperature by sputtering process and patterned by a lift-off process. Next a low-temperature $\left(100^{\circ} \mathrm{C}\right)$ deposition of ALD $\mathrm{ZnO}$ was carried out using 250 ALD cycles. ${ }^{8}$ The deposition temperature was optimized to get the desired film resistivity that allows TFT operation. Often higher deposition temperatures lead to higher conductivity in $\mathrm{ZnO}$, hindering effective control of the carrier concentration in the channel via gate modulation. This leads to transistors which turn ON under zero gate bias (depletion mode transistors) and attain low $\mathrm{I}_{\text {on }} / \mathrm{I}_{\text {off }}$ ratio. ${ }^{7}$ The film resistivity was confirmed by a four-point probe measurement to be $\sim 1 \Omega \mathrm{cm}$. Next, device isolation was carried out by wet etching using diluted $\mathrm{HF}\left(\mathrm{H}_{2} \mathrm{O}: \mathrm{HF}=25: 1\right)$ solution for $10 \mathrm{~s}$. A $4 \mu \mathrm{m}$ thick photoresist was used to protect $\mathrm{ZnO}$ active area. Fig. 1(e) shows a $50 \mu \mathrm{m}$ channel length transistor, with 16 fins. The figure is for a bottom gate bottom contact device, and it shows an overlap area between the $\mathrm{ZnO}$ and Ti-Au electrodes.

The planar device has a device width of $250 \mu \mathrm{m}$ and gate length of $50 \mu \mathrm{m}$. In the fin design, as shown in Fig. 1(a), $\mathrm{W} 1=4 \mu \mathrm{m}$ and $\mathrm{W} 2=1.5 \mu \mathrm{m}$ were chosen as the fin width and height, respectively, achieving an aspect ratio of $\sim 3$. W3 was varied to vary the number of fins used per-device, and $\mathrm{W} 3=4,8,12$, and $20 \mu \mathrm{m}$ giving 32, 21, 16, and 10 fins per transistor occupying the same chip area of the planar device. The extra device width, $W_{\text {extra }}$, due to the fin architecture can be calculated as

$$
W_{\text {extra }}=2 * W 2 * \text { number of fins per device. }
$$

This would give $W_{\text {extra }}$ of $30,48,63$, and $96 \mu \mathrm{m}$ for the fabricated 10, 16, 21, and 32 fin devices, respectively.

A cross sectional scanning electron microscope (SEM) of the $\mathrm{ZnO} / \mathrm{Ti}-\mathrm{Au} / \mathrm{Al}_{2} \mathrm{O}_{3} / \mathrm{Si}$ is shown in Fig. 2(a) showing a 


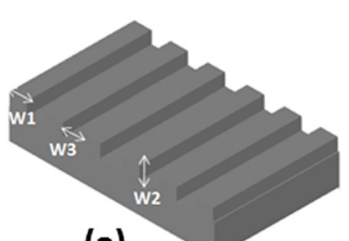

(a)

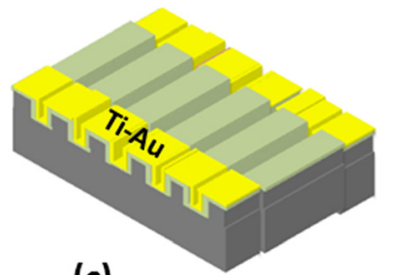

(c)

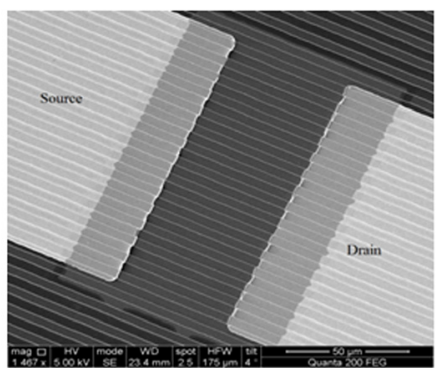

(e)
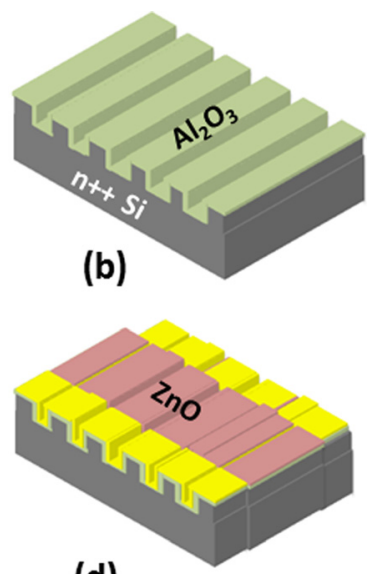

(d) fin features in the heavily doped n-type substrate, where $\mathbf{W} \mathbf{1}$ is the fin width, $\mathbf{W 2}$ is the fin height, and $\mathbf{W 3}$ is the distance between two consecutive fins. This is followed by (b) ALD Deposition of $\mathrm{Al}_{2} \mathrm{O}_{3}$ gate dielectric, (c) Ti-Au direct current (DC) sputtering of the source and drain and patterning them by lift-off process, and finally (d) deposition of ALD ZnO and device isolation by dilute HF solution. (e) SEM image showing top view of a 16 fin $50 \mu \mathrm{m}$ channel fabricated TFT.

thickness of $\sim 47 \mathrm{~nm}$ of $\mathrm{Al}_{2} \mathrm{O}_{3}, 128 \mathrm{~nm}$ of Ti-Au layer, and $\sim 40 \mathrm{~nm}$ of $\mathrm{ZnO}$, respectively. The films are uniform and conformal without any voids. The SEM image also shows a smooth interface with both the gate dielectric and the source and drain metal pads. The Grazing Incidence X-Ray Diffraction (GIXRD) confirms that the film is mostly amorphous with very weak Wurtzite peaks, as shown in Fig. 2(b), and Fig. 2(c) shows an Atomic Force Microscopic (AFM) image of the ZnO film. The SEM image (Fig. S1 in supplementary material ${ }^{14}$ ) shows nano-sized grains in $\mathrm{ZnO}$ film further confirming GIXRD finding.

The transfer and output characteristics of the planar and wavy channel TFTs are compared and shown in Figs. 3(a) and 3(b), respectively. An early turn-on of the devices as well as an increased drain current is noticed as the number of fins increases. Threshold voltages, $\mathrm{V}_{\mathrm{t}}$, are 6.4, 3.0, 3.8, 2.6, and 2.1 for the planar, 10, 16, 21, and 32 fin devices, respectively. Threshold voltages were extracted using an extrapolation method in the saturation region that determines $V_{t}$ from the gate voltage axis intercept of the $\sqrt{\mathrm{I}_{\mathrm{d} s a t}}-\mathrm{V}_{\mathrm{g}}$ curve extrapolated at the point of its maximum first derivative, as reported in the literature. ${ }^{9}$ They are plotted against the number of fins in Fig. 4(a), where $V_{t}$ values are shown to decrease linearly as a function of the number of fins. For the drain current, the drain current value for the planar device is comparable to other reports in the literature for ALD based ZnO TFTs. ${ }^{10-13}$ As for the drain currents for the fin devices, they are shown also as a function of the number of in Fig. 4(b). The devices have shown a monotonic and linear increase in the drive current as a function of the number of fins showing a $3.7 \times$ increase over the planar devices for the same biasing conditions for the drain and gate for the largest number of fins, 32 fins. However, although the devices are showing linear increase of the drain current as a function of the number of fins incorporated, the increase in the drive current cannot be attributed only to the extra device width, $W_{\text {extra }}$, since for the 32 fins based TFT the ratio of the areas of the fin to the planar TFT is $\sim 1.4$ while the drain currents ratio is 3.7 , for the same biasing conditions as shown in Fig. 4(c). As a result, the $I_{o n} / I_{\text {off }}$ ratios of the wavy channel TFTs are higher than planar devices, which are of the order of $10^{5}$, considering an off current value of $1 \mathrm{nA}$ for all devices. Finally, we have checked for signs of $\mathrm{ZnO}$ channel delamination for wavy channel devices after electrical stressing using SEM imaging, and no signs of delamination or pinholes in the channel were observed. We have added this image as Fig. S2 supplementary material. ${ }^{14}$
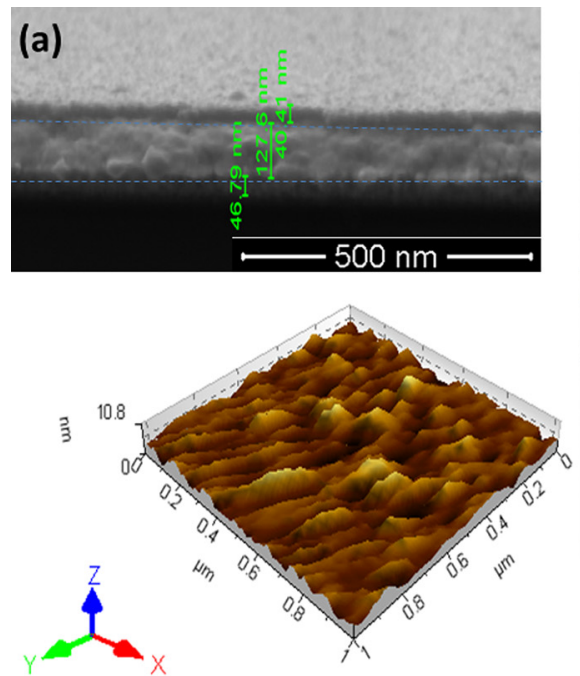

(c)

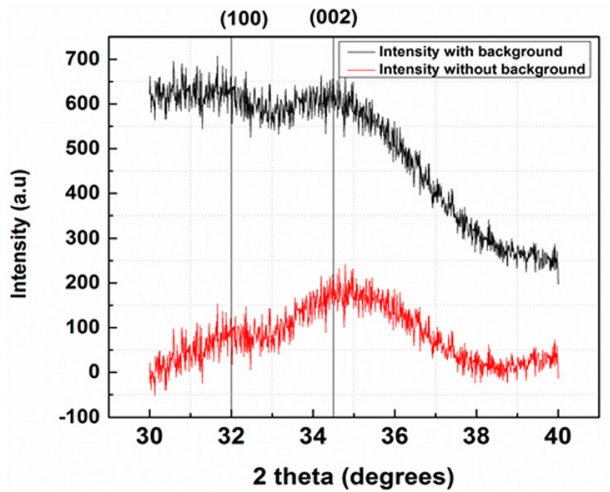

(b)
FIG. 2. (a) Cross sectional SEM showing the thicknesses (top to bottom) of the $\mathrm{ZnO}$, Ti-Au, and $\mathrm{Al}_{2} \mathrm{O}_{3}$ layers; (b) grazing incidence XRD with and without background, showing very weak peaks and proving the amorphous nature of $\mathrm{ZnO}$; and (c) an AFM image showing root mean square (RMS) roughness of $1.5 \mathrm{~nm}$. 


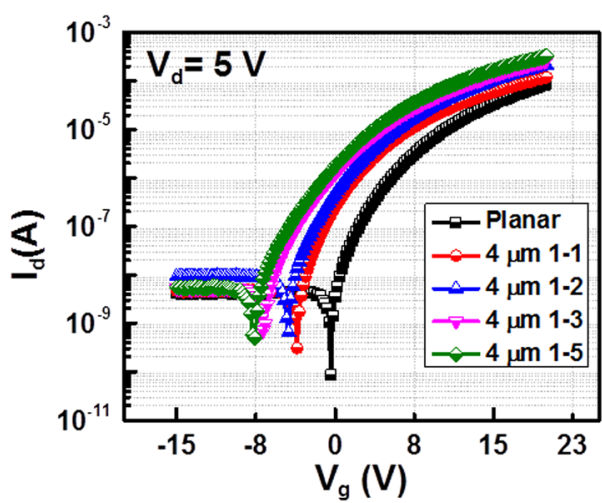

(a)
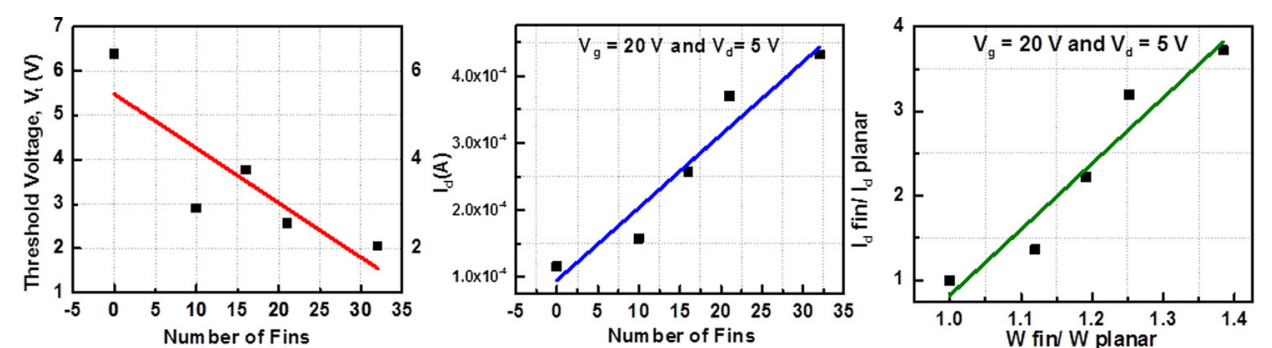

FIG. 3. (a) Transfer and (b) output characteristics comparison between fin TFTs and planar transistor allconsuming the same chip area. The convention for naming fin devices is $W 1$ 1: $\frac{W 3}{W 1}$. So $4 \mu \mathrm{m} \mathrm{1:1}$ is a $4 \mu \mathrm{m}$ wide fin with $4 \mu \mathrm{m}$ distance between every two consecutive fins.
The increased device current can be explained on an enhanced electric field effect. In Fig. 5, the electric field lines around corners show an enhanced field due to field components from both the side walls of the fins and the planar part.

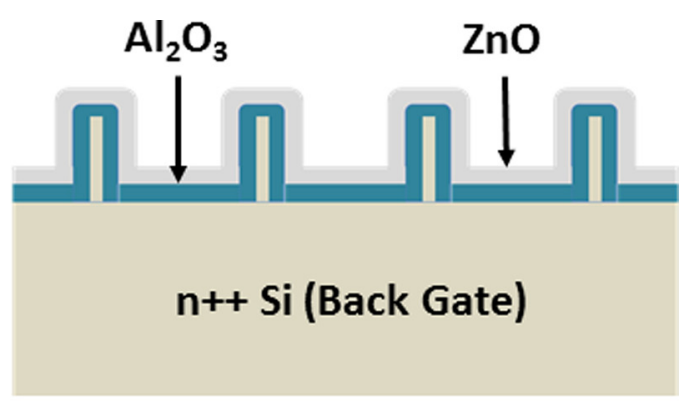

(a)

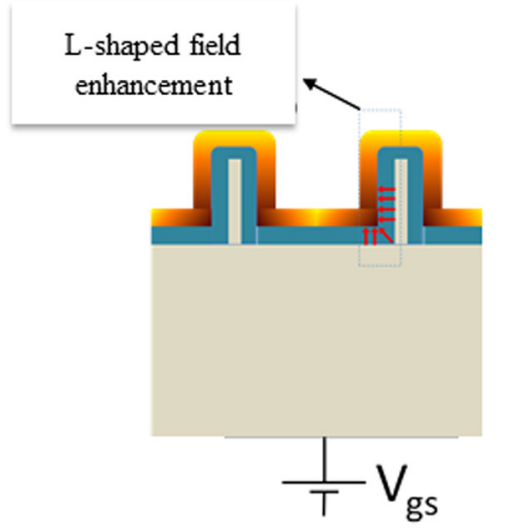

(b)

FIG. 5. Fin devices at (a) no bias and (b) with bias showing an enhancement in the applied field at corners due to contribution from both the side walls and the planar part, which is termed L-shaped field enhancement.
We give this the term L-shaped field enhancement. This has been manifested in the devices as the threshold voltage decreased as a function of the number of fins as was shown in Fig. 4(a). To confirm our hypothesis, we have studied the current improvement for larger width, smaller aspect ratio: $16 \mu \mathrm{m}$ fins. Consistent results were found confirming that current improvement could be explained based on L-shaped field enhancement effect and suggesting that a higher current could be achieved using larger number of fins, or higher aspect ratio fins. We added the $16 \mu \mathrm{m}$ fins electrical characteristics to Figures S3 and S4 in supplementary material. ${ }^{14}$ The L-shaped field enhancement effect allows operation at lower voltages since control over the threshold voltage is possible through variation of the number of fins. This is advantageous since the threshold voltage can be tailored using the fin architecture to suit the operating voltage. That could also lead to more energy efficient since power consumption is lower when lower operating voltage $\left(\mathrm{V}_{\mathrm{dd}}\right)$ is used as power consumption (P) is quadratically proportional to the operating voltage: $\mathrm{P} \alpha$ $\mathrm{V}_{\mathrm{dd}}{ }^{2}$. This would allow the TFT to be integrated in applications that require low $\mathrm{V}_{\mathrm{dd}}$ such as active matrix organic light emitting diode (AMOLED) based screens for cell phones.

In conclusion, we have shown that wavy channel architecture thin film transistor allows expansion of device width in the vertical direction using the fin architecture. This architecture also allows the control over the threshold voltage as a function of the number of fins, which helps to tailor the TFT for lower operating voltages. The maximum number of fins device has shown a $3.7 \times$ increase of drive current over the planar counterpart consuming the same chip area. The enhancement was shown to be a linear function of the number of fins and cannot be attributed only to the extra vertical device width. An enhanced electric field effect is shown due to edges, namely, the L-shaped field enhancement. This could explain the earlier turn on of the devices as a function 
of the number of fins. Future work should examine fins of smaller fin width and larger fin height to examine their effects on both the drive current and switching behavior.

This work was supported in part by the KAUST Office of Competitive Research Funds (OCRF) under Grant CRG-1-2012-HUS-008.

${ }^{1}$ X. Guo, R. Sporea, J. Shannon, and S. R. Silva, ECS Trans. 22(1), 227 (2009).

${ }^{2}$ O. Bonnaud, P. Zhang, E. Jacques, and R. Rogel, ECS Trans. 37(1), 29 (2011).

${ }^{3}$ M. Bohr, Tech. Dig. - Int. Electron Devices Meet. 2011, 1.1.1-1.1.6.

${ }^{4}$ M. M. Hussain, C. E. Smith, R. Harris, C. Young, B. Sassman, H.-H. Tseng, and R. Jammy, IEEE Trans. Electron Devices 57(3), 626 (2010).

${ }^{5}$ M. Ritala, J. Niinistö, A. Jones, and M. Hitchman, Chemical Vapor Deposition (The Royal Society of Chemistry, Liverpool, 2009), p. 161.

${ }^{6}$ E. Fortunato, P. Barquinha, and R. Martins, Adv. Mater. 24(22), 2945 (2012).
${ }^{7}$ P. F. Carcia, R. S. McLean, and M. H. Reilly, Appl. Phys. Lett. 88(12), 123509 (2006).

${ }^{8}$ B. Oh, Y. Kim, H. Lee, B. Kim, H. Park, J. Han, G. Heo, T. Kim, K. Kim, and D. Seo, Semicond. Sci. Technol. 26(8), 085007 (2011).

${ }^{9}$ A. Ortiz-Conde, F. J. Garcia Sanchez, J. J. Liou, A. Cerdeira, M. Estrada, and Y. Yue, Microelectron. Reliab. 42(4), 583 (2002).

${ }^{10}$ S. K. Park, C. Hwang, H. Y. Jeong, H. Y. Chu, and K. I. Choa, Electrochem. Solid-State Lett. 11(1), H10 (2008).

${ }^{11}$ S. J. Lim, S. Kwon, H. Kim, and J.-S. Park, Appl. Phys. Lett. 91(18), 183517 (2007).

${ }^{12}$ C. C. Liu, Y. S. Chen, M. L. Wu, and J. J. Huang, ECS Trans. 11(5), 17 (2007).

${ }^{13}$ G. Gutierrez-Heredia, I. Mejia, N. Hernandez-Como, M. E. Rivas-Aguilar, V. H. Martinez-Landeros, F. S. Aguirre-Tostado, B. E. Gnade, and M. Quevedo, Low Temperature $\mathrm{ZnO}$ TFTs Fabrication with Al and AZO Contacts for Flexible Transparent Applications (Mater. Res. Soc. Symp. Proc., Boston, USA, 2012), Vol. 1494, p. 299.

${ }^{14}$ See supplementary material at http://dx.doi.org/10.1063/1.4836235 for the pictorial depiction of the SEM images of the $\mathrm{ZnO}$ wavy channel transistor, no delamination in $\mathrm{ZnO}$ film, electrical characteristics supporting the hypothesis of L-shaped field enhancement. 